\title{
A Staged Approach to Erosion Analysis of Wind Turbine Blade Coatings
}

\author{
David Nash ${ }^{1, *}$, Grant Leishman ${ }^{1}{ }^{1}$, Cameron Mackie ${ }^{2}$, Kirsten Dyer ${ }^{2}$ and Liu Yang ${ }^{1}$ \\ 1 Department of Mechanical \& Aerospace Engineering, University of Strathclyde, 75 Montrose Street, \\ Glasgow G1 1XJ, UK; grant.leishman@strath.ac.uk (G.L.); 1.yang@strath.ac.uk (L.Y.) \\ 2 Offshore Renewable Energy Catapult, Offshore House, Albert Street, Blyth NE24 1LZ, UK; \\ cameron.mackie@ore.catapult.org.uk (C.M.); kirsten.dyer@ore.catapult.org.uk (K.D.) \\ * Correspondence: d.nash@strath.ac.uk
}

Citation: Nash, D.; Leishman, G.;

Mackie, C.; Dyer, K.; Yang, L. A

Staged Approach to Erosion Analysis of Wind Turbine Blade Coatings. Coatings 2021, 11, 681. https: / / doi.org/10.3390/coatings 11060681

Academic Editor:

Alexander Tolstoguzov

Received: 15 May 2021

Accepted: 3 June 2021

Published: 5 June 2021

Publisher's Note: MDPI stays neutral with regard to jurisdictional claims in published maps and institutional affiliations.

Copyright: (C) 2021 by the authors. Licensee MDPI, Basel, Switzerland. This article is an open access article distributed under the terms and conditions of the Creative Commons Attribution (CC BY) license (https:// creativecommons.org/licenses/by/ $4.0 /)$.

\begin{abstract}
The current wind turbine leading-edge erosion research focuses on the end of the incubation period and breakthrough when analysing the erosion mechanism. This work presented here shows the benefits of splitting and describing leading-edge erosion progression into discrete stages. The five identified stages are: (1) an undamaged, as-new, sample; (2) between the undamaged sample and end of incubation; (3) the end of incubation period; (4) between the end of incubation and breakthrough, and (5) breakthrough. Mass loss, microscopy and X-ray computed tomography were investigated at each of the five stages. From this analysis, it was observed that notable changes were detected at Stages 2 and 4, which are not usually considered separately. The staged approach to rain erosion testing offers a more thorough understanding of how the coating system changes and ultimately fails due to rain droplet impacts. It is observed that during microscopy and X-ray computed tomography, changes unobservable to the naked eye can be tracked using the staged approach.
\end{abstract}

Keywords: wind energy; blades; leading-edge erosion; coatings; microscopy; X-ray computed tomography; staged analysis

\section{Introduction}

Wind turbine blade leading-edge erosion is a major issue within the wind energy sector [1,2]. Eroded blades perform at reduced aerodynamic efficiency, leading to a lower energy harvest and, in turn, less revenue for the wind farm owner [3-5]. Additionally, the maintenance cost of repairing an eroded blade can become very expensive due to the requirement of transport and technicians-this cost multiplies when considering offshore wind turbines. It is therefore important to know how a coating system is likely to fail, and a better understanding of this mechanism could then provide an estimate of when it is likely to fail.

Current methods for the erosion assessment of new coating systems involve a pass/fail test [6-8]. No in-depth analysis of the coating system through erosion progression has been conducted. A staged approach to erosion testing provides greater detail on how the coating system is changing with progressive rain impacts [9]. A staged approach also allows for time parameters to be assigned to each erosion stage. These time parameters can be linked to real-world applications and give an estimate of time to the end of incubation/failure of an operational coating system. However, it is noted that this does not include other real-world influential factors such as environmental conditions.

In a paper by Gaudern [10], a staged approach to erosion classification was developed but not fully exploited. Gaudern used photographs from operational wind turbines to create typical erosion patterns at various stages of erosion. This showed erosion starting as small pits that advanced into gouges and then developed into coating removal. However, the main purpose of that study was to investigate the aerodynamic impact of erosion at these stages and not the material effects. This paper outlines a staged approach to erosion 
testing, which focuses on the material changes at each stage. A five-stage approach to erosion testing is proposed, with each stage representing a key phase in the erosion process.

\section{Stages of Erosion}

The current investigations into rain erosion usually focus on two main areas, the end of the incubation period and the total lifetime of the material [11]. This approach only examines a limited number of points during the total lifetime of the material. Examining the material at an increased number of points gives a clearer picture of the changes the material experiences and helps understand the damage mechanism. Five stages were chosen as a pragmatic balance of frequent checking without over checking, disturbing the erosion mechanism and allowing for any relaxation to build up.

The five stages chosen are shown in Figure 1. Stage 1 represents the time before the sample was tested, which is useful to determine if there are any pre-existing defects or voids below the surface and how these affect the material's ability to resist erosion. Stage 2 is halfway through the incubation period. At this time, there will be no damage visible on the surface of the sample, but it is interesting to see if there are any defects or voids that have grown or been initiated by the erosion process. Stage 3 is at the end of the incubation period, ideally just before the increase in erosion rate occurs. This shows the changes in the sample that lead to the significant increase in mass loss. It can be difficult to stop the testing exactly at the end of the incubation period; therefore, a cautious approach was taken to make sure the sample was tested before a significant mass loss occurs. Stage 4 is during the steady rate of mass loss to look at how the sample was changing during this time and where the mass loss was occurring. Finally, Stage 5 is classified as the point where the composite substrate is showing, known as breakthrough. At this point, the sample is damaged beyond repair, and the mass loss will no longer be consistent.

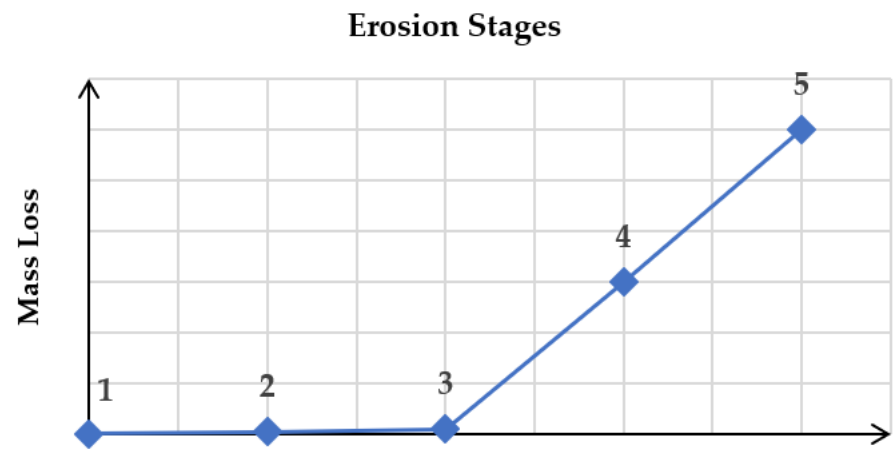

Time / Droplet Impacts

Figure 1. Erosion stages, typical mass loss plot.

Currently, standard industry practice only looks at Stages 1, 3 and 5, so by including Stages 2 and 4, a greater understanding of the transition can be determined. At Stage 2, the sample remains undamaged to the naked eye, but surface texture changes or sub-surface variations, such as volume of voids, can be detected using microscopy and /or X-ray computed tomography (XCT) [12]. Likewise, at Stage 4, increased volume of voids can be detected using $\mathrm{XCT}$, thus pointing towards a threshold value after which breakthrough results.

Throughout testing, the coated samples were characterised at the five different stages. The experimental measurements taken comprised photographs, microscopy, X-ray computed tomography and mass loss.

\section{Experimental Methodology}

The present work used a bespoke experimental test rig to explore different damage mechanisms to try and gain a better understanding of overall rig performance using a tightly controlled approach. The Whirling Arm Erosion test rig was designed, built and 
characterised at the Energy Technology Centre (ETC) in East Kilbride, UK [13]. ETC has been supporting the Offshore Renewable Energy (ORE) Catapult's erosion research programmes since 2015. The rig has 72 shrouded needles located at a single radius, with the ability to vary shroud height and run at up to $1400 \mathrm{rpm}$.

Single and double arm arrangements were initially experimented with to analyse whether the aerodynamic wake with two arms would be more detrimental than the benefit of two samples. It was discovered that, on this test rig, using just one arm produced more effective and repeatable results, as the aerodynamic wake produced by the arm influenced the air for approximately two-thirds of a rotation. Therefore, a second arm would induce turbulent air, thus making droplet formation and trajectory unpredictable, and for those reasons, a single-arm setup was considered optimal. Thereafter, an aerofoil was also attached to the arm to decrease the drag, which increases the stability of the droplets, allowing the arm to rotate at a faster rotational speed.

Examining different needle gauges, from 19 gauge to 30 gauge, determined which needles provided the most consistent diameter. A needle gauge of 27 was found to provide the most accurate droplet diameter over a range of pressures. Another improvement was to include plastic shrouds to protect the droplets while they form on the needle, and through work with a high-speed camera at 40,000 frames per second, it was seen that the plastic shrouds increased the number of whole droplets forming and, in turn, the number of droplet impacts. Without the plastic shrouds, considerable premature droplet breakup was observed. Premature breakup causes the droplets to implode due to turbulent air and split into tiny droplets. This subsequently adds an element of uncertainty to the results as the effect of tiny droplets cannot be quantified as easily as fully formed droplets. This makes it difficult to accurately quantify fractions of droplets in observations.

The water flow rate is controlled using a pressure head from a water tank on the wall. To increase or decrease the flow rate, water is removed or added to the tank. This process is controlled using an ABB Pressure Sensor, which keeps the pressure constant throughout the experiment. The pressure sensor measures the pressure change due to the changing pressure head then the corresponding pressures are set to the correct head height. Finally, leg adjustment was added to the ETC rig to allow for varying standoff heights. The standoff height is defined as the distance between the tip of the needle and the centre of the leading edge of the sample, as shown in Figure 2.

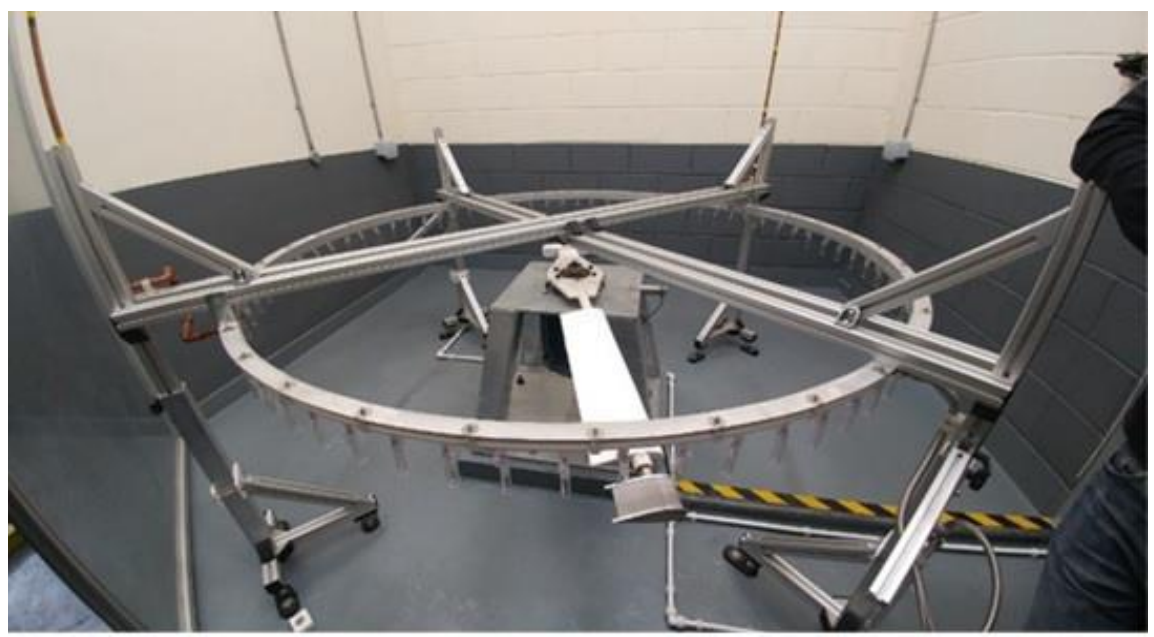

Figure 2. Rain Erosion Test (RET) rig at ETC, East Kilbride.

This previous work has led to the test rig being configured as shown in Figure 2. There is a single rotating arm that can spin up to $1400 \mathrm{rpm}$, with a tip speed of $150 \mathrm{~m} / \mathrm{s}$. Table 1 shows the conversion from rotational speed to linear speed. There are 72 needles spaced $5^{\circ}$ apart, mounted on a frame that can be adjusted to change the height of the needles, and the water pressure is determined by the pressure head on the tank, which is attached to the 
wall. The test rig can fit a variety of sample holders, both for aerofoil-shaped samples and flat samples.

Table 1. Rotational speed to linear speed of ETC rig.

\begin{tabular}{cc}
\hline Rotation Speed (RPM) & Linear Speed $(\mathbf{m} / \mathbf{s})$ \\
\hline 700 & 75.1 \\
800 & 85.9 \\
900 & 96.6 \\
1000 & 107.3 \\
1100 & 118.1 \\
1200 & 128.8 \\
1300 & 139.5 \\
1400 & 150.3 \\
\hline
\end{tabular}

The test rig was upgraded before the work started, with a hollow metal section replacing the PVC pipe manifold. This creates a more secure connection between the needles and the manifold as the needles are screwing into a sturdier material, which reduces the likelihood of leakage. Leaks can cause large droplets to fall and potentially impact the sample. This upgrade meant that the variation between the radii of needles is $2 \mathrm{~mm}$, which results in a very precise impact location.

Throughout the experimental testing, the test rig was operated at a rotational speed of $1200 \mathrm{rpm}$, with 36 of the needles operating. Calibration samples were used to validate each erosion stage and to provide an estimated run time in the test rig. The actual time taken to reach each of the erosion stages is shown below in Table 2.

Table 2. Time in RET rig to reach erosion stage for different test samples.

\begin{tabular}{cccccc}
\hline Erosion stage & A007 & A010 & A030 & A035 & A038 \\
\hline Stage 2 & - & $60 \mathrm{~min}$ & $60 \mathrm{~min}$ & $80 \mathrm{~min}$ & $60 \mathrm{~min}$ \\
Stage 3 & - & $100 \mathrm{~min}$ & $90 \mathrm{~min}$ & $120 \mathrm{~min}$ & $100 \mathrm{~min}$ \\
Stage 4 & $60 \mathrm{~min}$ & $110 \mathrm{~min}$ & $115 \mathrm{~min}$ & $140 \mathrm{~min}$ & $120 \mathrm{~min}$ \\
Stage 5 & - & $120 \mathrm{~min}$ & $120 \mathrm{~min}$ & $145 \mathrm{~min}$ & $130 \mathrm{~min}$ \\
\hline
\end{tabular}

The time taken to reach each stage is consistent, apart from sample A007, which failed unexpectedly, possibly due to a manufacturing defect or undetected initial void, reaching Stage 4 before any testing could be conducted on it at the previous stages. The test rig was initially stopped at $20 \mathrm{~min}$ intervals before decreasing to $10 \mathrm{~min}$ and then $5 \mathrm{~min}$ intervals as the erosion process accelerated.

The test specimens were manufactured using an arrangement of biaxial and unidirectional glass fibre mat, set in an epoxy resin using a vacuum infusion bag and mould technique. The glass fiber reinforced polymer substrate was then coated with the wind turbine blade leading edge coating system, according to the manufacturer's guidelines. Each specimen has three distinct layers-substrate, filler and Leading Edge Protection (LEP).

\section{Results}

Experimental results from the staged erosion testing method comprised photographs, microscopy images, $\mathrm{X}$-ray computed tomography $(\mathrm{XCT})$ slices and mass loss measurements. Note that the samples were not tested cumulatively; each erosion stage test had new samples. The exception to this is the XCT data, which were tested cumulatively on the same set of samples.

\subsection{Photographs of Samples at Five Stages of Erosion}

Images (Figure 3) were captured using the rear $12 \mathrm{MP}$ camera on a Samsung Galaxy S9 (Samsung, Seoul, Korea). 


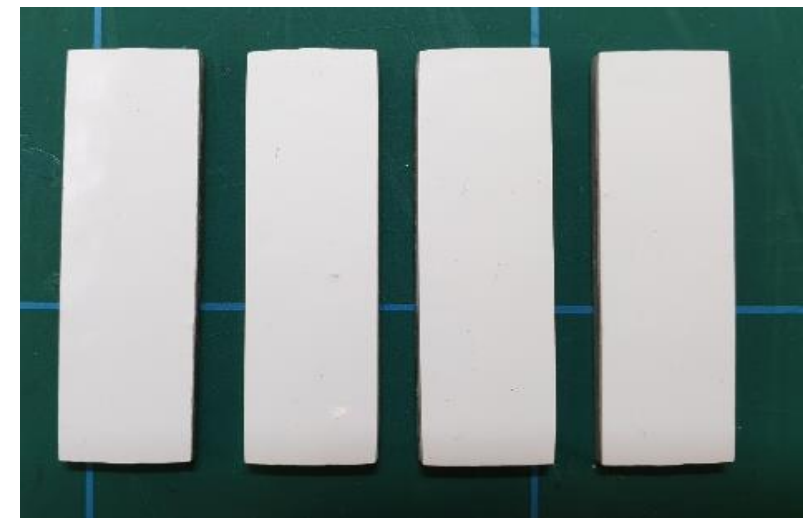

(a)

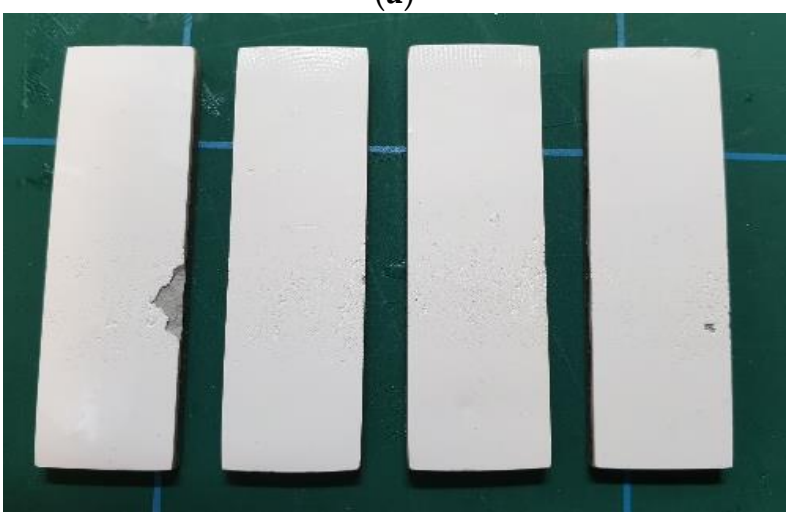

(c)

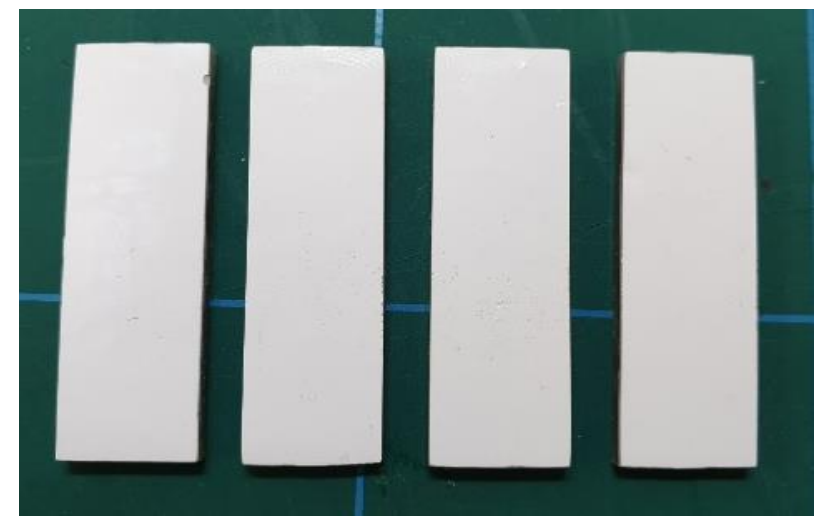

(b)

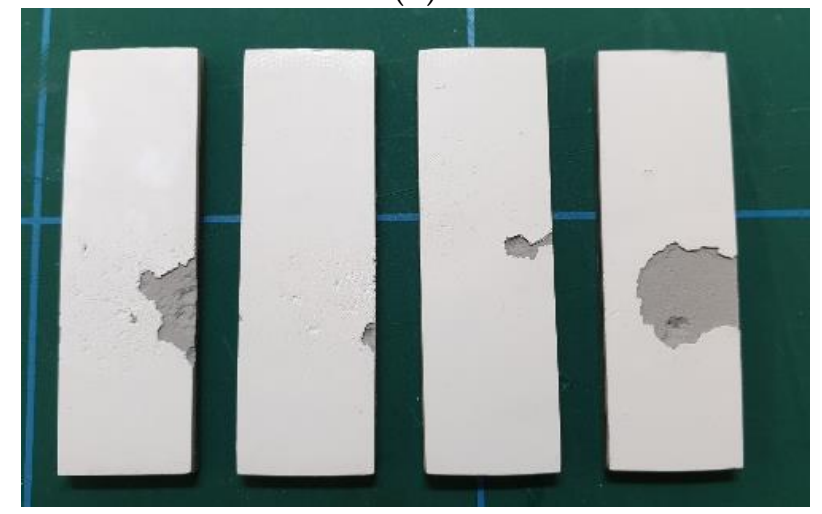

(d)

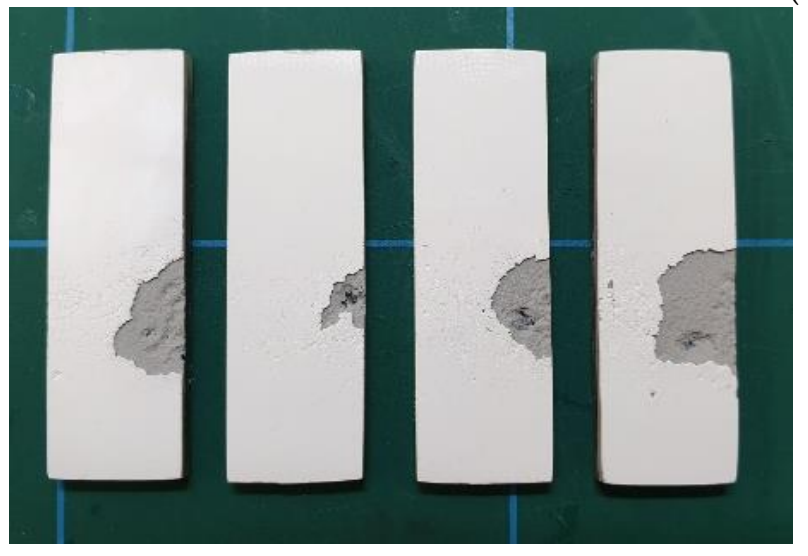

(e)

Figure 3. Photographs of samples from each erosion stage: (a) Stage 1; (b) Stage 2; (c) Stage 3; (d) Stage 4; (e) Stage 5.

\subsection{Microscopy of Samples at Five Stages of Erosion}

Images were captured using a Leitz Ergolux Optical Microscope (Leitz, Wetzlar, Germany) with a Leitz NPL FLUOTAR $10 \times / 0.22$ lens.

\subsection{X-ray Computed Tomography (XCT)}

A Nikon XT H 225 LC X-ray computed tomography system (Nikon, Tokyo, Japan) was used to generate the raw imagery. The raw imagery was then reconstructed using Nikon CT Pro software, yielding a voxel size of $14.8 \mu \mathrm{m}^{3}$. The reconstructed data were then processed, and the figures produced using Dragonfly software [14].

The void volume in each layer was measured using the XCT apparatus. The void volume was determined by measuring the total volume of each layer, and the Dragonfly software used can determine the amount of air in that given area. The void volume in the 
LEP layer is shown below in Table 3, and the void volume in the filler layer is shown below in Table 4.

Table 3. Voids in LEP layer.

\begin{tabular}{ccccc}
\hline Title & A010 & A030 & A035 & A038 \\
\hline Stage 1 & $0.38 \%$ & $0.36 \%$ & $0.71 \%$ & $0.27 \%$ \\
Stage 2 & $1.64 \%$ & $2.75 \%$ & $2.99 \%$ & $2.84 \%$ \\
Stage 3 & $1.37 \%$ & $6.30 \%$ & $4.81 \%$ & $3.44 \%$ \\
Stage 4 & $6.30 \%$ & $11.09 \%$ & $6.97 \%$ & $7.62 \%$ \\
Stage 5 & $30.66 \%$ & $36.78 \%$ & $12.78 \%$ & $56.10 \%$ \\
\hline
\end{tabular}

Table 4. Voids in filler layer.

\begin{tabular}{ccccc}
\hline Title & A010 & A030 & A035 & A038 \\
\hline Stage 1 & $0.63 \%$ & $0.92 \%$ & $0.69 \%$ & $0.52 \%$ \\
Stage 2 & $0.84 \%$ & $0.94 \%$ & $0.76 \%$ & $0.93 \%$ \\
Stage 3 & $1.83 \%$ & $1.14 \%$ & $1.31 \%$ & $1.03 \%$ \\
Stage 4 & $1.40 \%$ & $1.77 \%$ & $1.29 \%$ & $1.00 \%$ \\
Stage 5 & $4.52 \%$ & $4.65 \%$ & $3.11 \%$ & $8.22 \%$ \\
\hline
\end{tabular}

\subsection{Mass Loss during RET}

The mass of each sample was recorded using an A \& D Instruments GR-120-EC mass balance (A \& D, Tokyo, Japan). Once samples were tested using the RET rig, they were left at ambient conditions for a minimum of two weeks prior to mass loss measurement. This is to allow any retained moisture from the test to evaporate. Error bars represent a 95\% confidence limit on the results. Impact energy was calculated as the total kinetic energy of the water droplets striking the sample surface.

\subsection{Results Discussion}

This staged approach to rain erosion testing offers a more insightful look into the erosion process and allows for an assessment of the initiation and development of erosion. Stage 1, shown in Figure 3a, displays the coated sample before any rain erosion testing-a fresh, new sample. Stage 2, shown in Figure 3b, displays the sample after a period of rain erosion testing, where the sample has remained within the incubation period. Stage 3, shown in Figure 3c, displays the sample just as the incubation period has come to an end and the sample has begun to show signs of erosion. Stage 4, shown in Figure 3d, displays the sample during active erosion-the rate of material loss from the sample is at its highest during the erosion process. Stage 5, shown in Figure 3e, displays the sample where the coating material has completely failed and the composite substrate was revealed.

Looking at the coating system surface under a microscope at the various erosion stages, it is clear that there are distinct phases of surface texture according to each of the erosion stages. Figure 4a presents the coating surface at Stage 1. It should be noted that the surface is not perfectly smooth or uniform; air bubbles are present. The air bubbles could be due to the application method of the coating system or the mixing technique used in the coating preparation. However, to the naked eye, as in Figure 3a, no surface defects or air bubbles are visible. Moving to Stage 2, Figure $4 \mathrm{~b}$, there is a clear, noticeable surface change. The air pockets from Stage 1 have collapsed due to the impacting rain droplets; the air pocket sites have now developed into pits. As the sample is exposed to further rain droplets, these pits begin to grow and merge, as is observable from Figure $4 \mathrm{~b}$. 


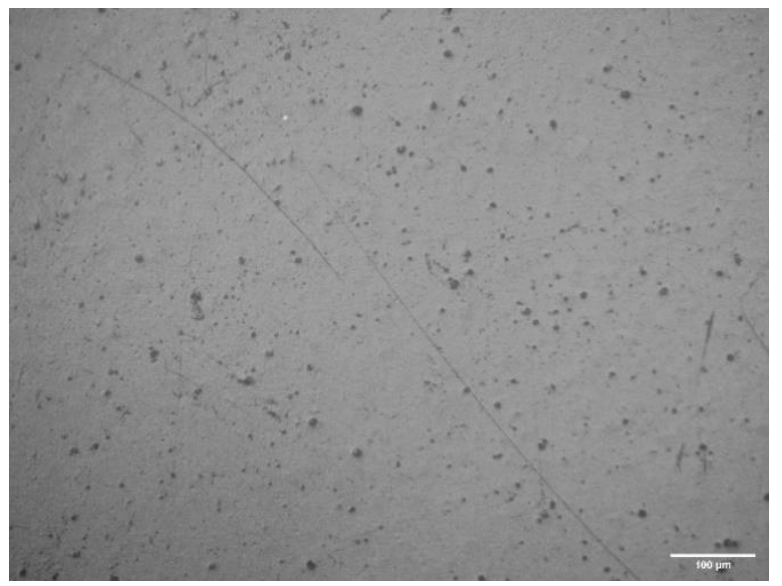

(a)

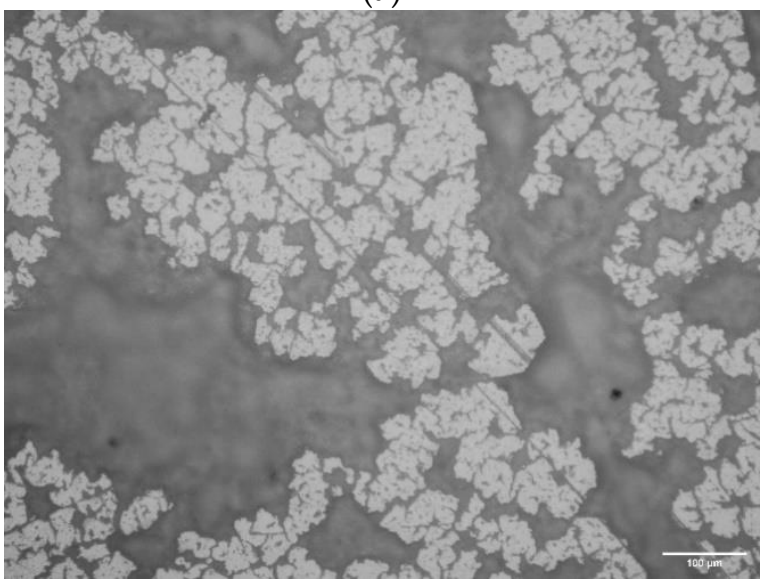

(c)

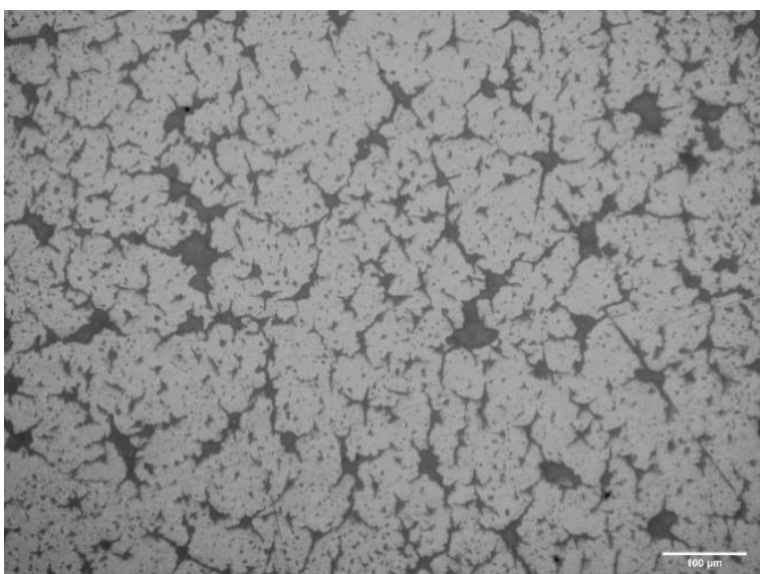

(b)

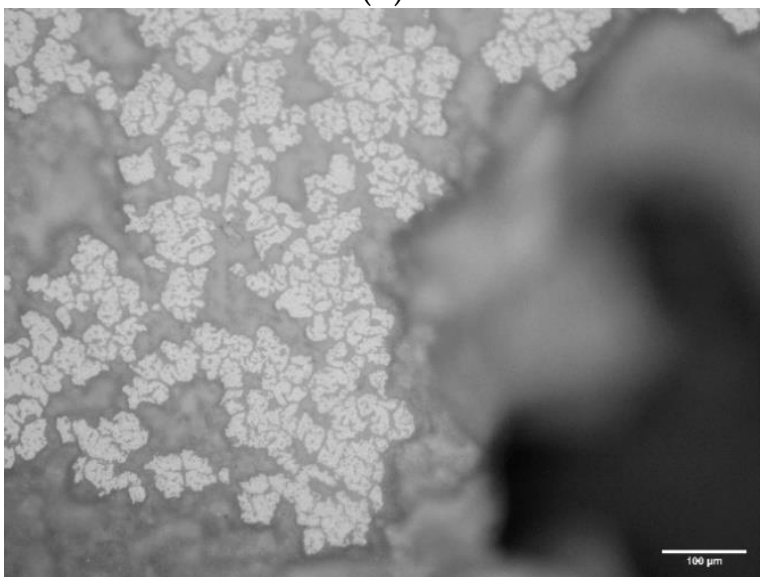

(d)

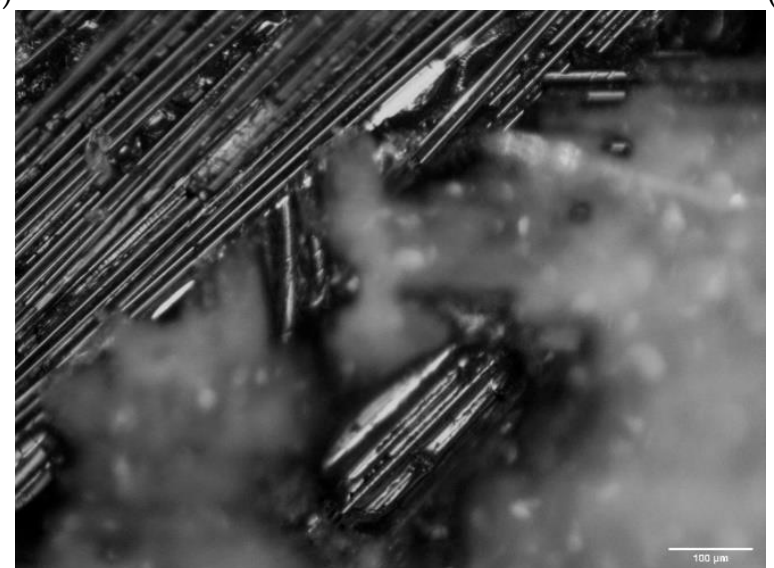

(e)

Figure 4. Microscopy images of samples from each erosion stage: (a) Stage 1; (b) Stage 2; (c) Stage 3; (d) Stage 4; (e) Stage 5.

It is important to note that this surface change is not visible to the naked eye, see Figure 3b. By using visual inspection and mass loss alone, the Stage 2 surface change would go unnoticed. Stage 2 is a crucial step as this is where the initial erosion damage begins to form; the surface changes from mostly smooth and uniform to ridged with small pits and gouges. These pits and gouges then act as the initiation site for further erosion damage. Looking at Stage 3 erosion, Figure 4c, the pits formed during Stage 2 have further developed and collated to produce channels in the coating surface. During Stage 3 erosion is where the effect of the rain droplet impacts starts to become visible to the naked eye, as seen in Figure 3c. This is also the stage where the mass loss of the sample begins to 
accelerate. Stage 3 is where a major structural change in the coating occurs. The channels in the coating act as initiation sites for further erosion and promote the progression of erosion on the existing damage.

Moving on to Stage 4 erosion, Figure $4 \mathrm{~d}$, the channels created during Stage 3 have deepened and further collated with nearby channels. This has led to the removal of sections of the coating from the sample. In the bottom right corner of Figure $4 \mathrm{~d}$, the dark area present is a large crater where the coating material was removed. When Stage 4 is reached, and the filler material is visible, the erosion area drastically deepens and widens due to the weak, porous structure of the filler. This leads to highly accelerated erosion. Finally, looking at Stage 5, Figure 4e, sections of the entire coating system were removed, exposing the fibreglass composite substrate. This is a result of the continued impacting droplets on the craters developed during Stage 4.

Observing the XCT data, three distinct layers are present. Starting at the bottom of Figure 5a, the glass fibres of the substrate are visible. The bright white layer on top of the substrate is the filler layer, and the thinner grey layer on top of the filler is the LEP. Stage 1, Figure $5 \mathrm{a}$, shows that voids are present within the coating system (filler and LEP) and the composite before any testing has begun. Progressing through Figure 5a-e, the erosion stages are clearly visible. The results of the void volume in the LEP layer, shown in Table 3, show that the void volume increases throughout the erosion process. This is to be expected with the coating as erosion occurs on this layer first, so by the time Stage 5 is reached, there can be a significant mass loss, which is shown by the large volume shown in Figure 5e. While the measurement of the voids in the LEP might be affected by erosion as early as Stage 2, the filler should remain undamaged until probably Stage 4 and definitely Stage 3.

Table 4 clearly shows there is an increase in void volume in the filler layer as the sample erodes. It can be seen at stages 1-3 (the filler will not have experienced any mass loss at this stage), there is a considerable increase. For samples such as A010, the void volume trebles from Stage 1 to 3, whereas for sample A030, there is only a small increase, but there is consistent evidence of an increase in void volume regardless of the sample. This is an interesting observation, as it should not be possible for new voids to be formed during the erosion process. This suggests that the original voids are somehow increasing in volume. One explanation for how this occurs is that tiny voids next to each other in the sample will merge together by multiple stress waves breaking down the small wall between them $[12,15]$. This will then increase the total volume measured. Another consideration is that the filler layer has some particulate matter present in it. These particulates could cause stress concentrations which lead to minute cracks forming, thus increase the total void volume. For most samples, there is a slight drop in the void volume at Stage 4, which will be due to the samples being measured in a different orientation for Stages 4 and 5 . Thus making it difficult to measure the same volume of the sample, as if a slightly smaller volume is measured, this could result in the anomaly in the results. 


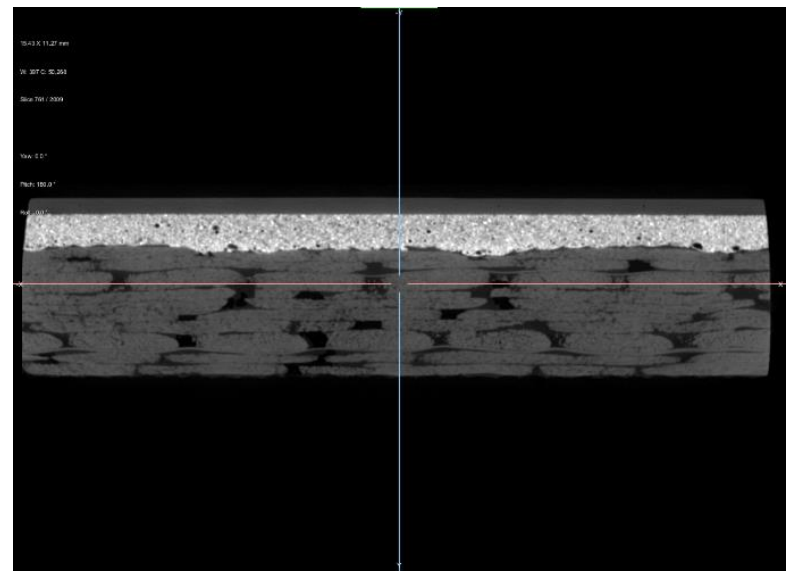

(a)

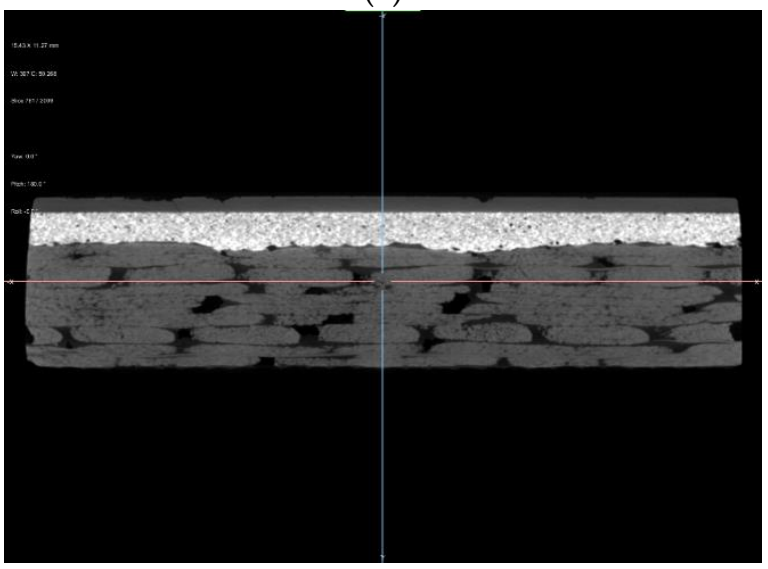

(c)

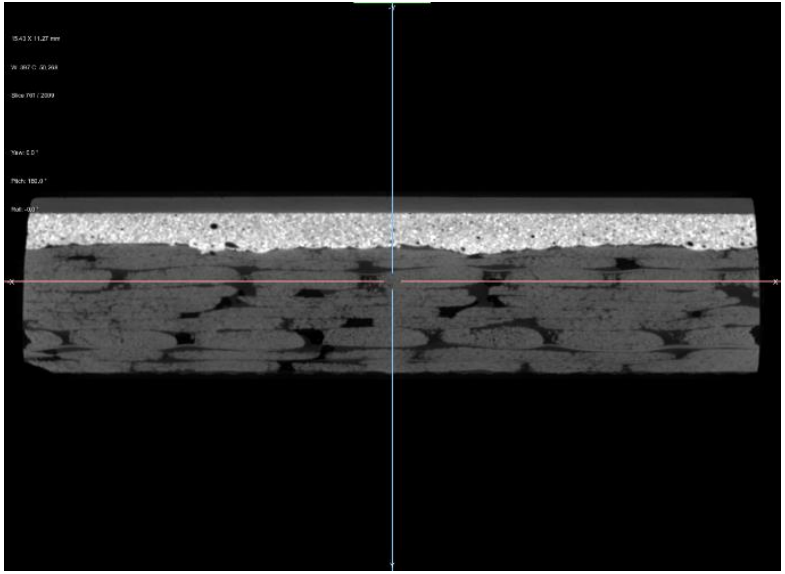

(b)

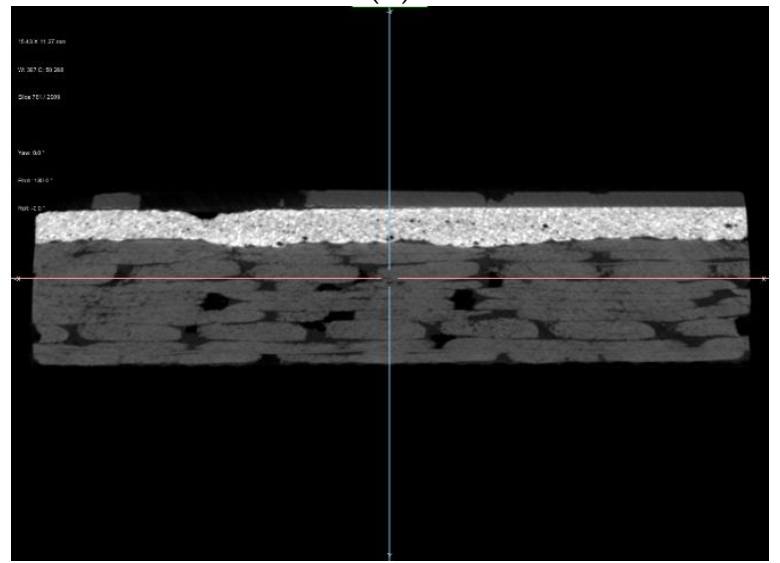

(d)

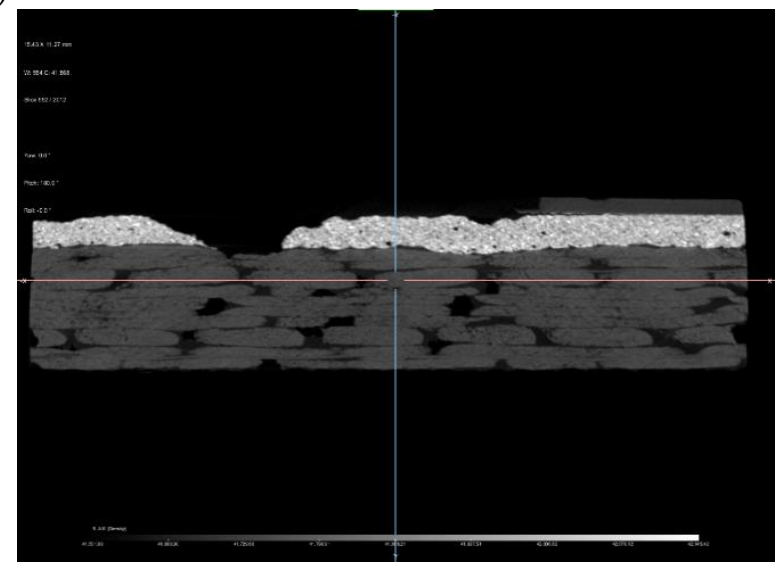

(e)

Figure 5. XCT images of samples from each erosion stage: (a) Stage 1; (b) Stage 2; (c) Stage 3; (d) Stage 4; (e) Stage 5.

Considering the mass measurements taken at each stage of erosion (Figure 6a) it is clear that there is no considerable loss in mass until after the incubation period has passed-once Stage 3 is reached. Beyond Stage 3, the erosion process accelerates, with greater mass loss observable at each erosion stage. Observing the plot of mass loss vs. impact energy (Figure 6b) the nature of the accelerated erosion from Stage 3 to Stage 5 is evident by the amount of impact energy it takes to go from Stage 3 to Stage 5-around 1200 Joules. Whereas the amount of energy required to reach Stage 3 is around 4400 Joules. This demonstrates that once Stage 3 erosion is reached, the coating system is rapidly progressing towards failure. Therefore, the most informative stage of the erosion process is between Stage 1 and Stage 3. 


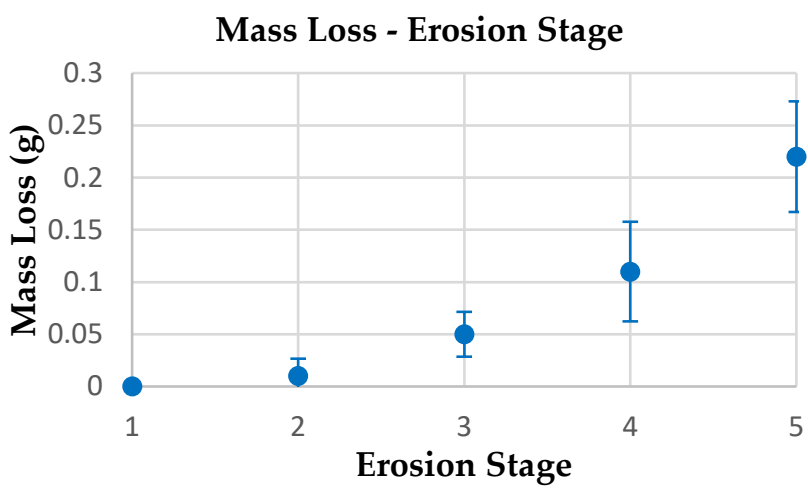

(a)

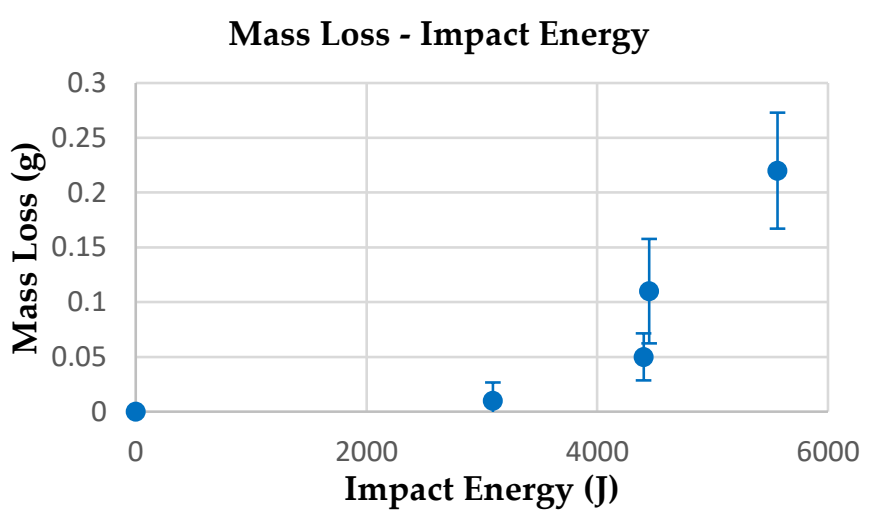

(b)

Figure 6. Plots of mass loss vs.: (a) Erosion Stage; (b) Impact Energy.

Looking at all the data presented, it is clear that the erosion is concentrated on a particular zone of the sample surface. This is due to the nature of the RET rig used. The ETC rig uses a repeated bombardment of rain droplets on a focused area of the sample and not across the whole sample surface, whereas other RET rigs may use different rain droplet layouts.

Additional to the data presented in this paper, a Fourier transform infrared spectroscopy (FTIR) (4100 ExoScan FTIR, Agilent Technologies, Santa Clara, CA, USA) study was undertaken to look at any change in the chemical bond groups within the coating's top surface, the LEP. This study found that as the erosion stages progressed during RET, no change in chemical bond groups was detected. This then suggests that the molecules which make up the coating system, the intramolecular bonds, are not breaking down. However, that the chemical bonds, which hold the molecules together, the intermolecular bonds, are breaking down. It is hypothesised that the persistent impact from rain droplets is leading to groups of molecules being removed from the LEP by breaking their intermolecular bonds. This then becomes evident when looking at Stage 2 erosion under the microscope, and the surface has begun to change.

\section{Conclusions}

A staged approach to rain erosion testing offers a more thorough understanding of how the coating system changes and ultimately fails due to rain droplet impacts. The experiments conducted herein show that there are five distinct phases of erosion-this is evident from an examination of the surface microstructure and the void volumes within each layer of the coating system. The five stages of the erosion model offer greater detail than the commonly used time to reach incubation and time until breakthrough to the substrate tests.

Using mass loss alone is not an accurate method of measuring erosion. The variability of results, shown by the error bars in Figure 6, suggests a combined approach of mass loss, photography and microscopy is required to accurately assess the erosion stage.

The results from the microscopy and XCT show that even when changes are unobservable to the naked eye, there can be significant changes to be tracked. These tools can be utilised to gain a greater understanding of the erosion process. The microscopy and XCT show that the assumption of no noticeable changes during the incubation period is incorrect. The staged approach model allows these changes to be explored further, thus providing a more complete picture.

The erosion stages identified in this work can be used during industrial testing of coating systems to provide greater detail on coating performance, allowing for greater certainty in coating lifetime prediction. 
Author Contributions: Conceptualization, D.N. and K.D.; methodology, D.N., G.L., C.M., K.D. and L.Y.; validation, D.N., G.L. and C.M.; formal analysis, G.L. and C.M.; investigation, G.L., C.M.; resources, D.N., K.D. and L.Y.; data curation, G.L.; writing-original draft preparation, G.L. and C.M.; writing-review and editing, D.N., G.L., C.M., K.D. and L.Y.; supervision, D.N. and K.D.; project administration, D.N. and K.D.; funding acquisition, D.N. All authors have read and agreed to the published version of the manuscript.

Funding: This research was part funded by EPSRC Centre for Doctoral Training in Wind and Marine Energy Systems grant number EP/L016680/1.

Institutional Review Board Statement: Not applicable.

Informed Consent Statement: Not applicable.

Data Availability Statement: Data is contained within the article.

Conflicts of Interest: The authors declare no conflict of interest.

\section{References}

1. Keegan, M.; Nash, D.; Stack, M. On erosion issues associated with the leading edge of wind turbine blades. J. Phys. D Appl. Phys. 2013, 46, 383001. [CrossRef]

2. Bartolomé, L.; Teuwen, J. Prospective challenges in the experimentation of the rain erosion on the leading edge of wind turbine blades. Wind Energy 2018, 22, 140-151. [CrossRef]

3. Mishnaevsky, L.; Hasager, C.; Bak, C.; Tilg, A.; Bech, J.; Doagou Rad, S.; Fæster, S. Leading edge erosion of wind turbine blades: Understanding, prevention and protection. Renew. Energy 2021, 169, 953-969. [CrossRef]

4. Han, W.; Kim, J.; Kim, B. Effects of contamination and erosion at the leading edge of blade tip airfoils on the annual energy production of wind turbines. Renew. Energy 2018, 115, 817-823. [CrossRef]

5. Koodly Ravishankara, A.; Özdemir, H.; van der Weide, E. Analysis of leading edge erosion effects on turbulent flow over airfoils. Renew. Energy 2021, 172, 765-779. [CrossRef]

6. ISO/TS 19392-1:2018. Paints and Varnishes_Coating Systems for Wind-Turbine Rotor Blades; BSI Standards Publication: London, UK, 2018.

7. ASTM G73-10. Standard Test Method for Liquid Impingement Erosion Using Rotating Apparatus; ASTM International: West Conshohocken, PA, USA, 2017.

8. DNVGL-RP-0171. Testing of Rotor Blade Erosion Protection Systems; DNVGL: Bærum, Norway, 2018.

9. Elhadi Ibrahim, M.; Medraj, M. Water Droplet Erosion of Wind Turbine Blades: Mechanics, Testing, Modeling and Future Perspectives. Materials 2019, 13, 157. [CrossRef] [PubMed]

10. Gaudern, N. A practical study of the aerodynamic impact of wind turbine blade leading edge erosion. J. Phys. Conf. Ser. 2014, 524, 012031. [CrossRef]

11. Herring, R.; Dyer, K.; Martin, F.; Ward, C. The increasing importance of leading edge erosion and a review of existing protection solutions. Renew. Sustain. Energy Rev. 2019, 115, 109382. [CrossRef]

12. Mishnaevsky, L.; Fæster, S.; Mikkelsen, L.; Kusano, Y.; Bech, J. Micromechanisms of leading edge erosion of wind turbine blades: X-ray tomography analysis and computational studies. Wind Energy 2019, 23, 547-562. [CrossRef]

13. Mackie, C.; Nash, D.; Boyce, D.; Wright, M.; Dyer, K. Characterisation of a Whirling Arm Erosion Test Rig. In Proceedings of the 2018 Asian Conference on Energy, Power and Transportation Electrification (ACEPT), Singapore, 30 October-November 2018.

14. Dragonfly 2020.2 [Computer Software]; Object Research Systems (ORS) Inc.: Montreal, QC, Canada, 2020. Available online: http:/ / www.theobjects.com/dragonfly (accessed on 23 February 2021).

15. Mishnaevsky, L.; Sütterlin, J. Micromechanical model of surface erosion of polyurethane coatings on wind turbine blades. Polym. Degrad. Stab. 2019, 166, 283-289. [CrossRef] 